\title{
Modelo para avaliar o comportamento dinâmico da evolução da comercialização de produtos em um contexto de inovação aberta
}

\section{Model to evaluate the dynamic behavior of the evolution of product marketing in an open innovation context}

ISSN 0104-530X (Print) ISSN 1806-9649 (Online)

\author{
Eugenio Andrés Díaz Merino ${ }^{1}$ \\ Fernando Antônio Forcellini' \\ Rafael Ariente Neto ${ }^{2}$ \\ Adriano Wagner ${ }^{1}$
}

\begin{abstract}
Resumo: Esta pesquisa é estabelecida no contexto da inovação aberta. Nesse contexto, a abertura do processo de inovação acarreta fatores específicos que podem comprometer seu desempenho. Assim, o objetivo geral desta pesquisa consistiu em evidenciar a influência de dois fatores na taxa de comercialização de produtos ao longo do tempo: (i) a geração de conhecimento externo e (ii) a entrada de novos concorrentes. Esta pesquisa descritiva utiliza a Dinâmica de Sistemas resultando no diagrama de causalidade e no diagrama de fluxo e estoque no tratamento qualitativo e quantitativo. É evidenciado que a geração de conhecimento externo pode influenciar a abertura de novos mercados conduzindo ao aumento de consumidores potenciais e efetivos. A entrada de novos concorrentes influência o sistema pelo valor no mercado e seu reflexo na percepção do consumidor. Isto se expressa em uma taxa de comercialização alongada e um crescimento abreviado de unidades comercializadas.
\end{abstract}

Palavras-chave: Gestão da inovação; Inovação aberta; Dinâmica da inovação; Dinâmica de Sistemas; Comportamento dinâmico; Modelo de avaliação.

\begin{abstract}
This research is set in the context of open innovation. In this context, the opening of the innovation process entails specific factors that can compromise its performance. Thus the main objective of this research was to demonstrate the influence of two factors on the rate of commercialization of products over time: (i) the generation of external knowledge and (ii) the entry of new competitors. This descriptive study uses the System Dynamics resulting in the causal diagram and the flow and stock diagram in qualitative and quantitative treatment. It is shown that the generation of external knowledge can influence the opening of new markets leading to increased actual and potential consumers. The entrance of new competitors in the system influences the market value and its impact on consumer perception. This is expressed as a rate of elongated marketing and short growth of units sold.
\end{abstract}

Keywords: Innovation management; Open innovation; Dynamics of innovation; System Dynamics; Dynamic behavior; Evaluation model.

\section{Introdução}

A adaptação das organizações aos avanços cumulativos de tecnologia em termos globais, impulsionada pela competividade do ambiente mercadológico, culminou em uma gama de diferentes modos para geração de valor, bem como do próprio valor gerado. Essa adequação a uma necessidade e a obtenção de vantagens na exploração do mercado caracteriza o fenômeno da inovação para as organizações. A adoção ou prática da inovação nas empresas pode ocorrer no produto ou no processo em diferentes amplitudes

interna e/ou externamente para um mercado nacional ou mundial (Tálamo \& Carvalho, 2010; Sanches \& Machado, 2014). É inclusive apontada como um dos principais determinantes do aumento de produtividade de um país (CNI, 2009).

A abertura do processo de inovação para o ambiente externo à empresa caracteriza a "inovação aberta". Assim, a dinâmica dessa inovação ocorre em um contexto específico, podendo desencadear fatores dinâmicos que influenciam o comportamento do

\footnotetext{
${ }^{1}$ Departamento de Engenharia de Produção, Universidade Federal de Santa Catarina - UFSC, Campus Universitário, Trindade, CEP 88040-900, Florianópolis, SC, Brasil, e-mail: merino@deps.ufsc.br; forcellini@deps.ufsc.br; adrianowagner76@yahoo.com.br

${ }^{2}$ Departamento de Engenharia Mecânica, Universidade Federal de Santa Catarina - UFSC, Campus Universitário, Trindade, CEP 88040-900, Florianópolis, SC, Brasil, e-mail: rafael.ariente@gmail.com
} 
ciclo de vida do produto. As abordagens de análise e avaliação do ciclo de vida auxiliam a identificar as possibilidades de intervenção ao longo do ciclo de vida e as opções de melhora neste. Decisões relacionadas, por exemplo, ao volume de produção e às estratégias de comunicação podem ser tomadas apoiadas por técnicas de avaliação da evolução do ciclo de vida do produto.

Contudo, com a abertura do processo de inovação, dois principais fatores confrontantes atuam na dinâmica do ciclo de vida do produto no mercado, sendo: (i) a geração externa do conhecimento e (ii) a entrada de novos concorrentes. Nesse sentido, essa pesquisa descritiva parte de um problema de ordem gerencial que pode ser formalizado como: em um contexto que contempla a abertura do processo de inovação fatores dinâmicos específicos, conjuntamente aos efeitos de realimentação, defasagens e não linearidades podem condicionar o comportamento do sistema e, consequentemente, ocasionar decisões gerenciais errôneas caso não sejam considerados nas técnicas de análise.

Essa consideração se faz importante para a gestão de sistemas complexos (como o sistema em estudo), pois o fluxo de informações pode produzir comportamentos não lineares difíceis de serem controlados e conduzidos. Um comportamento desejável para o sistema pode ser descrito por conveniência a essa pesquisa como "o aumento da taxa de comercialização de produtos ao longo do tempo". Portanto, o objetivo geral desta pesquisa consiste em: evidenciar a influência da (i) geração de conhecimento externo e da (ii) entrada de novos concorrentes na taxa de comercialização de produtos ao longo do tempo. Com isso, e observada a importância da utilização de modelos para a compreensão dos fenômenos, os objetivos específicos deste estudo delineiam um processo de modelagem. Consistem em (i) construir requisitos orientados à abordagem do negócio que orientem a exploração do fenômeno da inovação aberta; (ii) descrever o sistema conceitualmente determinando os principais fatores de influência na dinâmica e os ciclos de realimentação atuantes; e (iii) propor um modelo que permita a simulação computacional para análise quantitativa do comportamento dinâmico do sistema. Para tal, um processo cognitivo estruturado é adotado para a construção dos requisitos estruturais. Já, para a descrição conceitual e específica para a simulação, utilizam-se as ferramentas da metodologia Dinâmica de Sistemas (DS).

Este trabalho foi planejado para apresentar contribuições em duas esferas, relativas ao resultado e ao processo. Em termos de resultados, contribui por (i) suprir uma lacuna específica, propondo um modelo para tratamento do fenômeno; (ii) identificar e organizar os fatores em um contexto permitindo uma interpretação do contexto de inovação aberta; e (iii) apresentar uma análise quanto ao comportamento desses fatores nesse contexto específico. Já em termos de processo, esse trabalho contribui por utilizar um processo estruturado como auxílio ao início do processo de modelamento. Portanto, pode oferecer suporte tanto para gestores como referência à análise $\mathrm{e}$ adoção de ações em um contexto de inovação aberta, como também pode apoiar estudantes e pesquisadores constituindo um exemplo de um processo de transformação da informação para construção de um modelo pela metodologia da Dinâmica de Sistemas.

Para tal, a estrutura desta apresentação do estudo compreende, além desta introdução, uma segunda seção que expõe e descreve os métodos e ferramentas. Também uma terceira seção que expõe as informações de fundamentação teórica sobre os assuntos utilizados no estudo; uma quarta seção que expõe as considerações realizadas e os resultados obtidos nas atividades de construção do modelo. Já a quinta seção inicia pelos testes aplicados para a confiabilidade do modelo e expõe e analisa os cenários projetados, fundamentando as conclusões da pesquisa constantes na sexta seção. A apresentação do estudo explicita as referências bibliográficas em sua sétima seção.

\section{Aspectos metodológicos}

A pesquisa é caracterizada quanto aos principais aspectos metodológicos conforme o apresentado no Quadro 1. Essa classificação é seguida pelo detalhamento do método de realização da pesquisa, bem como pela apresentação das ferramentas utilizadas.

Quadro 1. Caracterização metodológica da pesquisa.

\begin{tabular}{|c|c|c|l|}
\hline \multicolumn{2}{|c|}{ Aspecto } & \multicolumn{2}{c|}{ Classificação } \\
\hline (i) & Natureza do objeto & Descritiva & $\begin{array}{l}\text { Descreve os fatores mais importantes. No entanto, também } \\
\text { apresenta característica exploratória quando trata o fenômeno. }\end{array}$ \\
\hline (ii) & Natureza da pesquisa & Indutiva & $\begin{array}{l}\text { Estruturação predominantemente indutiva, mas com } \\
\text { característica dedutiva na interpretação. }\end{array}$ \\
\hline (iii) & Coleta de dados & Secundária & Obtida de bases bibliográficas e literatura especializada. \\
\hline (iv) & Abordagem do problema & Qualitativa & $\begin{array}{l}\text { Determinando e relacionando os principais fatores do sistema, } \\
\text { mas também qualitativa na etapa final de tratamento do } \\
\text { problema. }\end{array}$ \\
\hline (v) & Resultados & Aplicada & Orienta as ações para a gestão do processo. \\
\hline
\end{tabular}

Fonte: Elaborado pelos autores. 


\subsection{Materiais e métodos}

O método de pesquisa pode ser representado por cinco etapas principais como é ilustrado na Figura 1. Essa perspectiva geral também expõe previamente os meios para operacionalização de cada etapa.

Definição do problema: A pesquisa tem foco no problema descrito inicialmente, contudo a própria realização da pesquisa prova a expansão da compreensão deste problema. Nesse sentido, as etapas do método geram conhecimento cumulativamente e a evolução dos modelos mentais permite uma compreensão expandida dos diferentes aspectos do problema abordado. Isto porque esses modelos mentais referem-se à visão de mundo de cada indivíduo, para cada caso. São constituídos de uma mistura de compreensão e valores reunidos ao longo de suas experiências e de sua educação (Jackson, 2007). Os modelos mentais determinam a forma de pensar ou a estrutura de análise de um caso.

Caracterização: É o início do processo de modelagem nessa pesquisa. Estabelece as bases fundamentais do modelo direcionando a estruturação e delimitação do estudo. Essa caracterização é realizada por um processo cognitivo estruturado. A primeira atividade deste processo consiste em selecionar um referencial teórico que possibilite a delimitação e caracterize o modelo. Com base nesse referencial, são identificadas lentes que especificam as facetas pelas quais o problema será analisado. Em outros termos, visto que nesse estágio inicial são vastas as possibilidades de abordagem do problema, as lentes especificam "o que procurar" (os elementos de análise) de modo assegurado pelo embasamento. A seleção de um referencial teórico e a identificação de lentes neste processo de interpretação são realizadas objetivando a viabilização da construção cumulativa do conhecimento por utilizar as bases fundamentais em uma metodologia já existente, bem como facilita a compreensão por empregar uma linguagem já conhecida. O foco de cada lente especifica "onde procurar" nos elementos da análise. Esse processo de interpretação cognitiva estruturada é representado na Figura 2. No contexto sistêmico da pesquisa, esse processo estruturado utilizado para a caracterização do modelo objetiva reduzir o nível de abstração ao qual a etapa de estruturação está sujeita, mesmo com a descrição completa e ampla do problema.

Estruturação: Pelas bases fundamentais, o modelo é caracterizado permitindo a compreensão e análise. Por este estudo, tem interesse a interpretação do comportamento do estado de um sistema específico em um horizonte de tempo em um contexto em que atuam fatores conflitantes foram utilizadas as etapas e ferramentas da metodologia de Dinâmica de Sistemas. Com isso, os modelos construídos no desenvolvimento desta pesquisa vinculam-se à linguagem utilizada por essa metodologia. A DS pode ser utilizada em contextos não lineares com alto nível de abstração por uma linguagem versátil permitindo uma compreensão sistêmica, além da simulação e projeção de cenários do comportamento do sistema (Coyle, 1996). Sendo assim, a estruturação compreende tanto a descrição do sistema (ou modelagem qualitativa ou interpretativa ou conceitual), a modelagem estrutural e a modelagem matemática. A construção desse modelo é uma atividade de transformação da informação, sequencial ou interativa, contribuindo com a construção do conhecimento em diferentes aspectos. A descrição conceitual do sistema se utiliza do diagrama de causalidade. Consiste basicamente em uma lista de fatores de um problema, sendo que setas e sinais mostram o relacionamento entre estes fatores (Coyle, 1996). Enquanto esse diagrama descreve o sistema formalizando e delimitando o contexto, também aborda identificando e associando ciclos de realimentação que conduzem à informação. O conceito é detalhado pelo diagrama de fluxos e estoques que engloba as constantes e variáveis necessárias e suficientes, possibilitando a descrição matemática das relações.

Interpretação: Engloba desde as atividades de configuração e geração da visualização gráfica do comportamento dos indicadores de estado do sistema em um horizonte de simulação. Para tal, são utilizados programas específicos para a Dinâmica de Sistemas que implementam o cálculo numérico para resolução do modelo matemático. Portanto, permite interpretar resultados de testes conduzidos em um

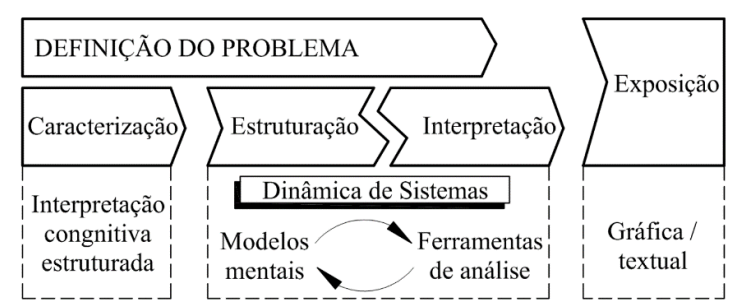

Figura 1. Método de pesquisa.

Fonte: Elaborado pelos autores.

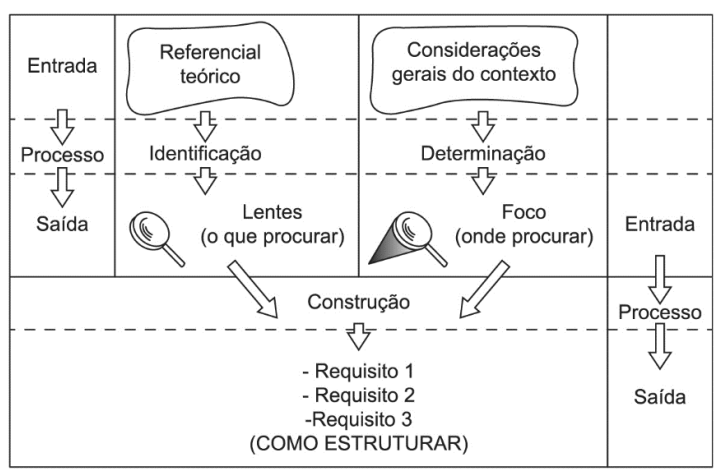

Figura 2. Processo de interpretação cognitiva estruturada. Fonte: Elaborado pelos autores. 
contexto virtual. Como os cenários são apresentados de modo gráfico, a etapa de interpretação estabelece uma nova atividade cognitiva de interpretação. Ainda, por afinidade dos pesquisadores e por possuir uma versão acadêmica gratuita, o software Vensim ${ }^{\circledR}$ (marca registrada de Ventana Systems Inc.) PLE (versão 6.2) foi utilizado. Outros softwares de modelagem pela linguagem da Dinâmica de Sistemas mais utilizados são: STELLA, Powersim e DYNAMO.

Exposição: É realizada textualmente em relação aos resultados obtidos e ao processo de obtenção desses resultados. Está organizada de modo estruturado e apresenta as principais conclusões da pesquisa.

\section{Fundamentação teórica}

A fundamentação teórica é conduzida de modo a abordar inicialmente os conceitos fundamentais que definem um modelo de negócio, seu conteúdo e utilidade. Posteriormente, é abordado o fenômeno da inovação no contexto mercadológico.

\subsection{A perspectiva do negócio}

De um modo amplo o modelo de negócio sob a ótica de uma organização pode ser compreendido como a representação de "um sistema de atividades interdependentes que transcende a organização focal e expande suas fronteiras" (Amit \& Zott, 2001). Além de esta definição abordar com uma perspectiva sistêmica o fenômeno do negócio, também considera que este pode estender-se para além das fronteiras de uma única organização (a organização focal) e abranger outros parceiros. Portanto, o modelo de negócio tem utilidade prática ao estudo dos fenômenos, envolvendo organizações por permitir em uma visão holística uma análise abrangente do valor na realização do negócio distinguindo a unidade organizacional, mas podendo incorporar aspectos referentes ao produto ou à rede de parceiros.

Por uma visão de processo (process thinking), a representação (modelo) do negócio engloba cadeias inteiras de eventos, atividades e decisões. Portanto, um processo de negócio consiste em "um conjunto de eventos, atividades e pontos de decisão inter-relacionados que envolvem uma série de atores e objetos e geram um resultado com valor para pelo menos um cliente" (Dumas et al., 2013). Essa visão é adotada para a representação de modelos de negócio (como modelos de processo de negócio) em diferentes linguagens de modelagem.

\subsection{Inovação no contexto mercadológico}

O conceito de inovação possui um caráter evolutivo e mostra-se contextualizado a segmentos de pesquisas, mas em termos técnicos a inovação pode ser vista como "um processo envolvendo pessoas e organizações criativas no qual um problema é estruturado e a informação é selecionada, integrada e ampliada gerando compreensão e respostas para o problema" (Batzias \& Siontorou, 2012). Pelo Manual de Oslo (OECD, 2007), a inovação no produto (bem ou serviço) ou no processo se efetiva quando novas tecnologias ou tecnologias substancialmente melhoradas são introduzidas no mercado (ou utilizadas no processo de produção para o caso dos processos). É pertinente salientar que uma inovação pode estar atrelada ao contexto da empresa (ou seja, o produto ou processo é novo para a empresa) ou a um contexto maior como o mercado em termos nacionais ou internacionais. Como aspectos comuns da inovação são apontados: resulta em novos produtos ou processos que diferem da condição anterior, demanda a exploração comercial como produto, processo, método, organização de trabalho ou relações externas (Chesbrough, 2006; OECD, 2010).

Dentre as diferentes manifestações no processo de inovação, a inovação aberta se destaca por, em um contrafluxo da estratégia comumente observada, buscar aporte externo para obter a diferenciação. Ou seja, a premissa básica da inovação aberta é a abertura do processo de inovação (Huizingh, 2011). Uma definição para a inovação aberta consiste em: "o uso direcionado de entradas e saídas de conhecimento para acelerar a inovação interna e expandir os mercados de uso externo da inovação, respectivamente" (Chesbrough et al., 2006).

\subsection{Inovação aberta na viabilização de experiências de realidade virtual}

Dentre as iniciativas de mercado atuais, um caso de inovação aberta a ser ressaltado consiste na condução do desenvolvimento do dispositivo de realidade virtual Oculus Rift pela empresa Oculus $V R^{\circledR}$ (Oculus VR 2014). A empresa arrecadou mais de \$2.4 milhões de dólares em fundos por meio do Kickstarter para o desenvolvimento de tal dispositivo que foi orientado para (i) ser um dispositivo para imersão em jogos, (ii) possuir interface simples e (iii) ser acessível financeiramente ao consumidor entusiasta de jogos.

Iniciativas anteriores, como o console da Nintendo "Virtual Boy" lançado no ano de 1995, tentaram explorar a realidade virtual para jogos, mas a tecnologia não proporcionava uma experiência imersiva sendo logo descontinuado. A primeira versão do Oculus Rift, denominada de Development Kit 1 (DK1) contava com uma visão de $3 \mathrm{D}$ estereoscópica (com resolução de $360 \times 480$ ), um campo de visão maior que $90^{\circ}$ (oferecendo inclusive uma visão periférica ao usuário) e uma baixa latência entre o movimento da cabeça e atualização da imagem (ao longo dos $360^{\circ}$ ) devido à utilização de sensores (giroscópio, acelerômetro e magnetos). Com essa associação de tecnologias, o protótipo DK1 conquistou muitos prêmios ao longo do ano de 2013 na categoria de dispositivos e hardwares. 
A versão DK1 foi disponibilizada para aquisição (direcionada a desenvolvedores e podendo incluir consumidores em geral) em março de 2013 pelo preço de $\$ 300.00$ (trezentos dólares), efetivando a abertura do processo de melhoria (ou inovação) do produto. A empresa especificou que não se tratava do produto final, portanto muitos materiais, tal qual a própria tecnologia, ainda não ofereciam a qualidade objetivada para a experiência do usuário. Foram vendidas 25 mil unidades em um mês, atingindo 60 mil unidades comercializadas desta versão. Essa abertura desencadeou reflexos evidenciados por compartilhamento de experiências. Dentre as quais: (i) o engajamento de desenvolvedores de jogos com o desenvolvimento de títulos específicos para a tecnologia e (ii) a exploração da tecnologia por desenvolvedores de aplicativos em segmentos distintos dos games. Nesta última, enquadram-se a possibilidade de viabilização de experiências em turismo virtual, aplicações terapêuticas como a exploração das fobias pela inserção virtual do paciente em situações controladas e seguras, como também um modo inovador de assistir a filmes. Ainda, (iii) a inclusão do próprio usuário no processo de obtenção de conhecimento, (iv) a realização de novas parcerias (com profissionais e empresas) e (v) o forte aumento da visibilidade do produto pelo compartilhamento prévio de experiências complementam tais reflexos. Ainda a recente fusão da empresa com o Facebook sugere que novas aplicações podem estar sendo desenvolvidas.

A segunda versão do Oculus Rift ainda como protótipo (o DK2) conta com melhorias tecnológicas substanciais. As melhorias de design do dispositivo, tornando-o mais atrativo e confortável, e as melhorias de resolução gráfica, evoluindo para maior qualidade de imersão em vários aspectos, possibilitando a abertura de amplas oportunidades de exploração da experiência vivida pelo usuário. A empresa desenvolvedora também ofereceu a opção de aquisição do software de desenvolvimento com código fonte aberto. Isso dá acesso à programação específica, acelerando o desenvolvimento de novos aplicativos.

Novos dispositivos com a mesma proposta também foram apresentados em eventos direcionados ao segmento, porém com menor visibilidade. Contudo, no evento E3 (da Entertainment Software Association) ocorreu a exposição do projeto da companhia Sony (o Project Morpheus) direcionado à exploração do segmento de realidade virtual em games. Por estar associado ao console PlayStation, preferido por muitos entusiastas do segmento, esse dispositivo representa um forte concorrente do Oculus Rift.

\section{Construção do modelo}

Esta seção apresenta a operacionalização do método de pesquisa e os resultados obtidos. Como ponto de partida para a construção do modelo, salienta-se que a questão fundamental que motivou a pesquisa, já apresentada na seção que concentra as informações introdutórias, consiste em: evidenciar as influências dos fatores dinâmicos específicos do contexto da inovação aberta no comportamento da taxa de comercialização de produtos ao longo do tempo. A explicitação novamente dessa questão é conveniente nesta etapa (de início do modelamento) por todas as atividades de modelagem e processos de transformação da informação estarem associados a ela.

Um comportamento desejável para o sistema pode ser descrito por conveniência a essa pesquisa como "o aumento da taxa de comercialização de produtos ao longo do tempo". Portanto, o objetivo geral desta pesquisa consiste em: evidenciar a influência da (i) geração de conhecimento externo e da (ii) entrada de novos concorrentes na taxa de comercialização de produtos ao longo do tempo. Com isso, e observada a importância da utilização de modelos para a compreensão dos fenômenos, os objetivos específicos deste estudo delineiam um processo de modelagem.

\subsection{Caracterização do modelo}

A seleção do referencial teórico para o processo de modelagem segue as linhas de interesse de investigação da pesquisa. Nesse sentido, adotou-se a definição do "modelo de negócio" conforme Dumas et al. (2013), sendo: "um conjunto de eventos, atividades e pontos de decisão inter-relacionados que envolvem uma série de atores e objetos e geram um resultado com valor para pelo menos um cliente". A definição do processo de negócio estabelece inteira convergência à consideração sistêmica. Expõe uma característica dinâmica, classifica os elementos do sistema de processo de negócio (diversos atores e objetos) e suas interações (conjunto de eventos, atividades e pontos de decisão inter-relacionados) e evidencia o objetivo conjunto (a geração de valor). Os aspectos evidenciados constituem, nesta pesquisa, as lentes do referencial teórico que efetivamente viabilizam a delimitação e caracterização do modelo, visto que orientam a construção de requisitos para o processo de estruturação.

- Elementos do sistema: atores e objetos que interagem na condução do processo de inovação aberta.

- Interações: eventos, atividades e pontos de decisão que estabelecem como ocorre a dinâmica do sistema.

- Objetivo: consideração em termos de valor do objetivo das ações do sistema.

As lentes ainda são complementadas pelo estabelecimento do foco para a construção dos 
requisitos. Para o estabelecimento do foco, buscou-se na bibliografia especializada (do ponto de vista dos pesquisadores) um conjunto comum de considerações que auxiliam a compreensão do contexto de modo amplo em que o problema se apresenta. Nesse sentido, a pesquisa ateve-se a informações que caracterizam e conceituam o fenômeno da inovação aberta. Conforme o fundamentado, as considerações que, nesta pesquisa (e, como tal, sob as perspectivas e interesses específicos), são selecionadas para a determinação dos focos das lentes um total de três considerações que consistem em:

- Abertura do processo de inovação - que caracteriza o fenômeno da inovação aberta (Huizingh, 2011; Chesbrough et al., 2006)

- Diferenciação do estado anterior - como a premissa básica para a condição de inovação (Lichtenthaler, 2011; Chiaroni et al., 2010)

- Abertura de novos mercados - como um resultado almejado com a ação da abertura do processo de inovação (Chesbrough et al., 2006; Enkel et al., 2009; Dahlander \& Gann, 2010).

Com o cruzamento das lentes com o foco, foram construídos os requisitos. Esses são representados por um verbo que caracteriza a ação para a estruturação do modelo. Os requisitos construídos são apresentados no Quadro 2, em que podem ser observadas lacunas. Nesse sentido, entendeu-se que deve haver uma aderência entre a lente e o foco.

Assim, os requisitos são construídos visando sua aplicação na estruturação do modelo e estabelecendo alinhamento com o referencial teórico são de ordem qualitativa (Jiao \& Tseng, 1999) não funcionais (Kotonya \& Sommerville, 2000; Labuschagne et al., 2005).
Sendo assim, impõem ressalvas ou restrições (nesse caso de ordem estrutural) para a modelagem do processo de negócio que engloba e permite analisar o fenômeno da inovação. A construção do modelo tem continuidade com a estruturação.

\subsection{Estruturação}

Estando a análise embasada e direcionada, neste estágio da pesquisa, se busca a expansão da compreensão sobre o contexto pela construção do diagrama de causalidade. Para tal, orientado pelos requisitos de ordem estrutural construídos na seção anterior, se busca a inserção das considerações específicas referentes à inovação aberta que estejam alinhadas para definir o contexto. Nesse sentido, o diagrama utiliza informações específicas.

Nessa pesquisa, a identificação dos fatores ocorre principalmente com fonte na bibliografia especializada e pela interpretação de um caso específico. Para a operacionalização dessa pesquisa, a identificação e a coleta de artigos foram realizadas utilizando a ferramenta "Google Acadêmico". Essa ferramenta viabiliza a obtenção de informações mais rapidamente pela facilidade de inserção de dados e filtragem temporal e de aspectos do conteúdo dos resultados, bem como efetua uma busca ampla englobando trabalhos de diferentes bases de dados (sendo o acesso à informação limitado ao acervo de bancos de dados disponíveis para acesso pela UFSC). Com isso, a atividade de coleta da bibliografia consistiu em uma primeira rodada de busca por termos de pesquisa. Os trabalhos coletados foram avaliados quanto ao seu alinhamento integral com o estudo, permanecendo os considerados pelos pesquisadores alinhados e com contribuição ao estudo. Posteriormente (na segunda rodada de busca), foram identificados e

Quadro 2. Relação de requisitos.

\begin{tabular}{|c|c|c|c|c|}
\hline \multirow{2}{*}{\multicolumn{2}{|c|}{ Lente }} & \multicolumn{3}{|c|}{ Foco } \\
\hline & & \multirow[t]{2}{*}{$\begin{array}{c}\text { Diferenciação do } \\
\text { estado anterior }\end{array}$} & \multirow{2}{*}{$\begin{array}{l}\text { Abertura do processo } \\
\text { de inovação }\end{array}$} & \multirow[t]{2}{*}{$\begin{array}{c}\text { Abertura de } \\
\text { novos mercados }\end{array}$} \\
\hline $\begin{array}{l}\text { Elementos do } \\
\text { sistema }\end{array}$ & Atores & & & \\
\hline & Objetos & & & \\
\hline \multirow{2}{*}{ Interações } & $\begin{array}{l}\text { Eventos/ } \\
\text { Decisão }\end{array}$ & & $\begin{array}{l}\text { (2) Associar a dinâmica } \\
\text { do sistema à abertura do } \\
\text { processo de inovação }\end{array}$ & $\begin{array}{l}\text { (3) Considerar a } \\
\text { abertura de novos } \\
\text { mercados }\end{array}$ \\
\hline & Atividades & & $\begin{array}{l}\text { (4) Considerar o } \\
\text { compartilhamento como } \\
\text { atividade do processo. }\end{array}$ & \\
\hline Objetivo & Valor & $\begin{array}{l}\text { (5) Direcionar a dinâmica } \\
\text { do sistema à diferenciação } \\
\text { do valor. }\end{array}$ & & \\
\hline
\end{tabular}

Fonte: Elaborado pelos autores. 
rastreados os trabalhos de referências utilizadas nas pesquisas identificadas anteriormente, obtendo-se novas fontes. Essas fontes também foram avaliadas quanto ao seu alinhamento integral com o estudo. Esse processo foi repetido até a composição de um portfólio. Conjuntamente, evidências empíricas foram extraídas do estudo de caso descrito anteriormente (no capítulo referente à fundamentação teórica) para complementar e compor o contexto.

Portanto, utilizando o termo de pesquisa "open innovation [and] management", "open innovation [and] managing", "open innovation [and] dynamics" e "open innovation [and] business" e "open innovation [and] model". Nesta primeira busca, os resultados foram analisados a partir do ano de 2010. Três trabalhos tiveram suas referências analisadas agregando ao conjunto de artigos de referência outros seis trabalhos. Em uma terceira rodada, em que as referências dos seis últimos artigos agregados ao portfólio foram analisadas, concluiu-se que os artigos alinhados integralmente à pesquisa já constavam do portfólio. Portanto, o Quadro 3 apresenta o conjunto de trabalhos adotados como referência.

A seguir, estão expostos e comentados os fatores (em forma de fragmentos conceituais específicos) identificados na literatura para a descrição contexto. Esses fatores consistem em:

- Entrada de conhecimento: conforme Tidd et al. (2005), a abertura do processo de inovação tem como um dos principais motivadores a obtenção de conhecimento do meio externo para aceleração desse processo.

- Conhecimento acumulado: ainda conforme Tidd et al. (2005), o acúmulo de conhecimento é um fator que conduz à efetivação da inovação. O conhecimento acumulado pode ser devido ao conhecimento gerado internamente na organização e, também, devido ao conhecimento externo coletado (Van de Vrande et al., 2009; Chiaroni et al., 2010).

- Aceleração da inovação interna: a inovação interna é acelerada ao passo que ocorre mais rapidamente a acumulação do conhecimento
(Chesbrough et al., 2006). Referente ao caso descrito para a realidade virtual, a significativa melhoria tecnológica funcional do dispositivo ocorreu em um horizonte de inovação aberta, portanto, nessa pesquisa, será relacionado à abertura do processo com a melhoria obtida. Assim, esse fator no contexto tem coerência com a bibliografia especializada e expõe que o conhecimento acumulado acelera o processo de inovação e resulta em melhorias substanciais do produto e aumentando, então, a percepção da qualidade na ótica do consumidor.

- Abertura de novos mercados: ressaltado pelas considerações de Chesbrough et al. (2006), este fator pode ser associado às observações do caso prático descrito. O desenvolvimento de novas aplicações para o produto conduz ao alcance de novos grupos de consumidores (como também se ressalta na descrição de caso). Ou seja, um aumento do número de potenciais consumidores para o produto. A abertura de novos mercados, no contexto desta pesquisa, também engloba as situações em que são encontrados novos meios externos para comercialização da inovação gerada internamente (Enkel et al., 2009; Dahlander \& Gann, 2010).

- Diferenciação do valor: no sistema descrito, a diferenciação do estado que caracteriza a inovação é exposta como a diferenciação do valor oferecido pelo sistema comparativamente a valores concorrentes no mercado (Lichtenthaler, 2011).

- Entrada de concorrentes: a abertura do processo de inovação pode acarretar a visibilidade pública dessa inovação (Huizingh, 2011). Com isso, não somente os desenvolvedores de novas aplicações podem ter acesso a essa inovação.

Esses fatores, portanto, são organizados em uma ótica sistêmica pelo diagrama de causalidade. As lacunas originadas ao longo da construção desse diagrama podem derivar da própria ausência de abordagens utilizando esta linguagem. Nesse caso, as lacunas

Quadro 3. Conjunto de trabalhos de referência.

\begin{tabular}{|c|c|}
\hline Primeira rodada (busca por termos) & Segunda rodada (busca pelas referências) \\
\hline Huizingh (2011) & Van de Vrande et al. (2009) \\
\cline { 2 - 2 } & Chesbrough et al. (2006) \\
\hline Dahlander \& Gann (2010) & Chesbrough \& Appleyard, (2007) \\
\hline Lichtenthaler (2011) & Chiaroni et al. (2010); \\
& Enkel et al. (2009); \\
& Chesbrough (2006). \\
\hline
\end{tabular}

Fonte: Elaborado pelos autores. 
foram supridas pela interação dos pesquisadores levando em conta seus próprios modelos mentais de entendimento do problema, evoluídos ao longo da pesquisa sem detrimento da confiabilidade do modelo. Nesse caso, eventuais relações errôneas podem ser identificadas na reprodução do comportamento do sistema já na atividade de simulação computacional (na etapa de testes). Assim, a construção do diagrama de causalidade engloba um trabalho de pesquisa indutiva, visto que identifica e conduz as informações para a descrição do contexto, e dedutiva no complemento das lacunas do diagrama. Como salientado durante toda a construção, foram observados os requisitos estruturais construídos na seção anterior. O diagrama de causalidade resultante é representado por inteiro na Figura 3.

A descrição conceitual do sistema pode ser interpretada com início no fator que determina a "abertura do processo de inovação", sendo que, um aumento da amplitude da abertura do processo de inovação acarreta um aumento do "compartilhamento de conteúdo". Conforme a definição de inovação aberta exposta por Chesbrough et al. (2006), a decisão de abertura do processo de inovação culmina em dois principais fatores: a aceleração da inovação interna e a expansão dos mercados externos. Ambos os fatores são viabilizados pelo conhecimento adquirido pela abertura do processo de inovação, contudo, a expansão do mercado é consequência da obtenção da inovação interna. No diagrama conceitual de causalidade o fator "aceleração do processo de inovação" é representado como efeito direto da "entrada de conhecimento". A "abertura de novos mercados" constitui um efeito indireto e sujeito à influência dos outros fatores do sistema descrito. A "diferenciação do valor" sintetiza, de modo geral, o objetivo do processo de inovação. É um efeito da "diferenciação do estado" proporcionada pela "aceleração da inovação interna" e do "conhecimento acumulado" pela abertura do processo de inovação. A maior "diferenciação do valor" leva a maior "satisfação dos consumidores" e, por consequência, a maior quantidade de "consumidores efetivados".

Com isso, são evidenciados pelo diagrama de causalidade dois principais ciclos de realimentação da informação que influenciam o comportamento dinâmico deste sistema de acordo com os fatores utilizados. Esses ciclos são: a "necessidade de conhecimento" (ciclo de reforço) e a "saturação do mercado" (ciclo de balanceamento); e são novamente abordados no detalhamento do sistema. Além dos ciclos de realimentação, a representação conceitual do sistema também indica os atrasos (ou defasagens) de tempo, sendo nomeados de D1 a D5. A identificação

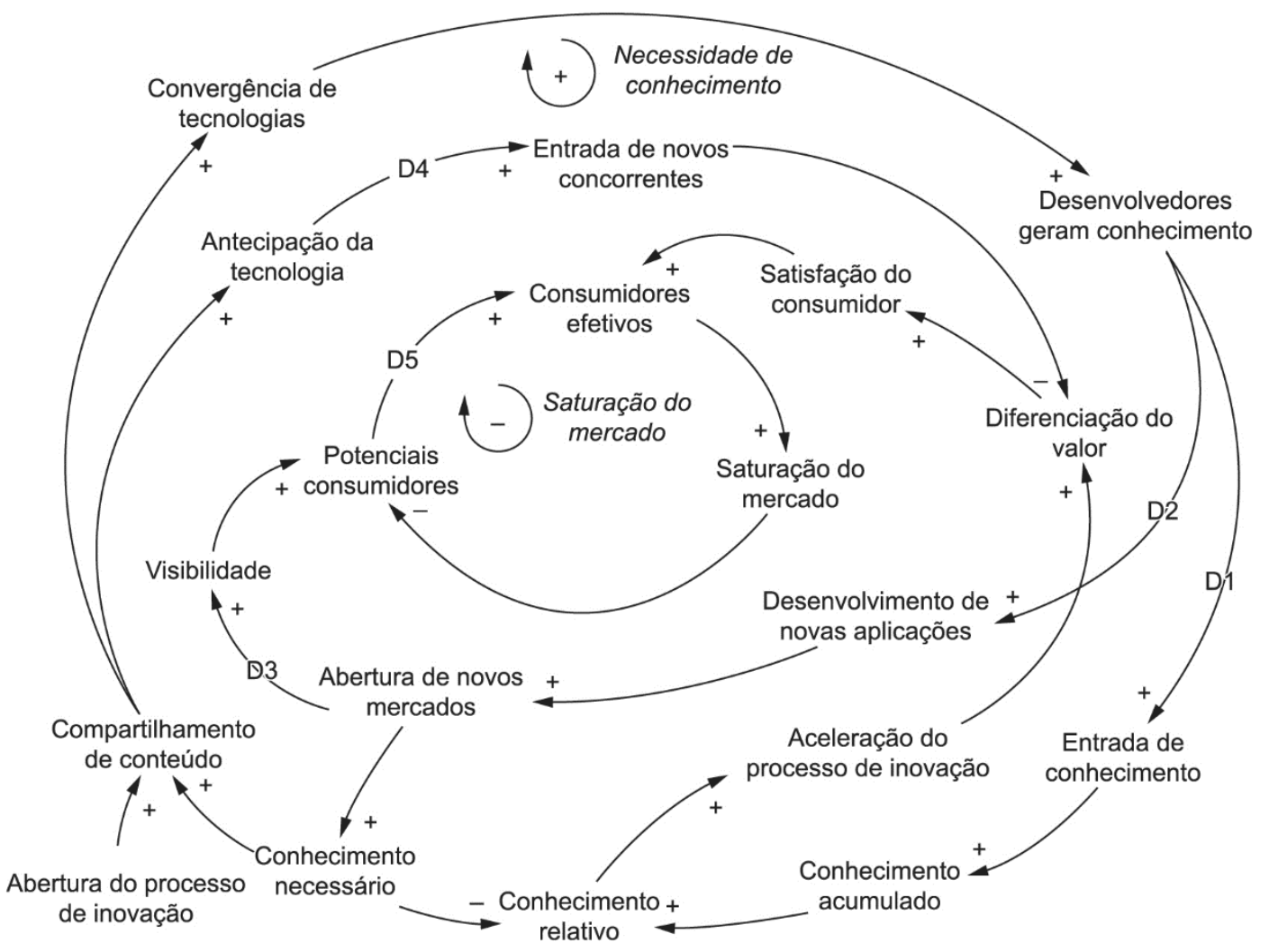

Figura 3. Diagrama de causalidade. Fonte: Elaborado pelos autores. 
desses atrasos auxilia na interpretação da dinâmica de atuação dos fatores.

As informações do modelo conceitual nessa continuidade do processo são traduzidas em uma linguagem que permite a descrição matemática das relações do contexto. A representatividade do diagrama de fluxo e estoque para a pesquisa está em manter os conceitos do diagrama de causalidade em uma linguagem que possibilita a simulação computacional. Uma vez que o comportamento consiste em uma representação resultante da relação entre as constantes e variáveis que compõem e definem um sistema, ou seja, sua estrutura (Sterman, 2000), o processo de construção do diagrama de fluxo e estoques teve como base a estrutura apresentada por Sterman (2000, p. 343). Essa estrutura insere, portanto, o aspecto do comportamento de "limitação ao crescimento" ou curva "S" e é complementada por constantes e variáveis de taxas e estoques que complementam o contexto conforme as considerações da dinâmica da inovação aberta. O emprego da estrutura genérica de gestão de estoques em pesquisas que analisam a evolução do ciclo de vida do produto também pode ser visto com Kazemi et al. (2011), que estabelece estratégias de gestão.
Com isso, esse diagrama, além dos principais ciclos de realimentação evidenciados na descrição conceitual, ainda contém os ciclos que compõem a estrutura genérica e ciclos estabelecidos à medida que o detalhamento é executado. A Figura 4 apresenta o resultado dessa atividade da pesquisa.

As defasagens de tempo indicadas no diagrama de causalidade são transcritas nas respectivas constantes. $\mathrm{Na}$ representação do diagrama de fluxo e estoques, essas constantes de tempos mantêm a nomenclatura utilizada no diagrama conceitual, sendo que sua influência no contexto detalhado fica evidenciada. Ainda, as variáveis "efeito do conhecimento compartilhado na entrada de concorrentes", assim como o "efeito do surgimento de novas aplicações na abertura de mercados" e os demais fatores correlatos que podem ser observados no diagrama de fluxos e estoques, simplificam o tratamento das relações de suas variáveis a montante e a jusante. No caso específico do "efeito do conhecimento compartilhado na entrada de concorrentes", assumiu-se uma relação linear, ou seja, assumiu-se, de modo geral, um aumento de conteúdo compartilhado, que causa uma redução proporcional no "tempo médio para a entrada de concorrentes". Do mesmo modo, para o "efeito do surgimento de novas aplicações na abertura de

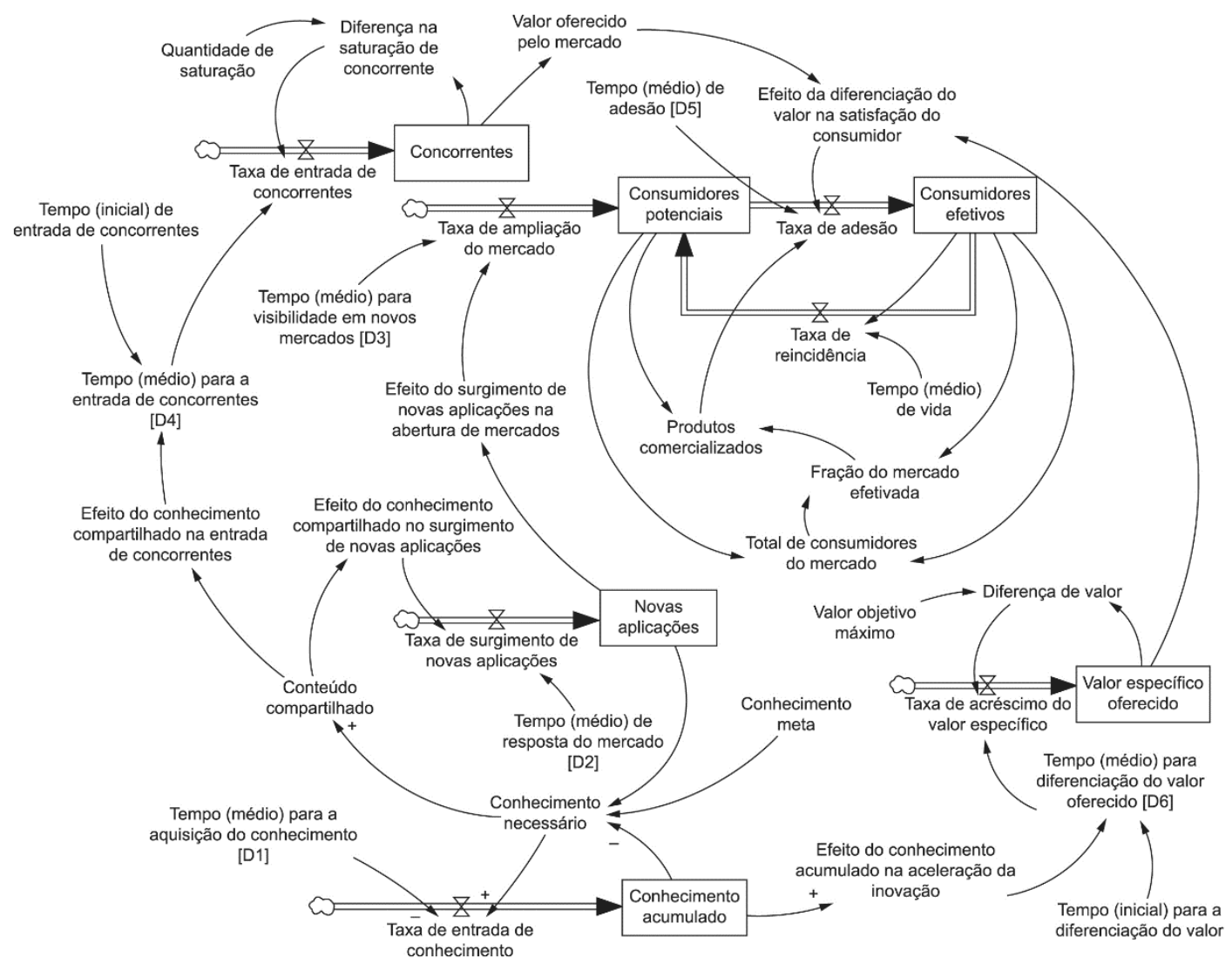

Figura 4. Diagrama de fluxo e estoques do sistema. Fonte: Elaborado pelos autores. 
mercados", assumiu-se que o surgimento de novas aplicações causa um aumento proporcional na taxa de ampliação do mercado.

Cada relação (representada pelas linhas que ligam os fatores, taxas e estoques) agrega uma descrição matemática e o conjunto de relações especifica como ocorre cada relação previamente descrita no diagrama conceitual. O processo de estabelecimento dessas relações corresponde ao modelamento matemático. O diagrama de fluxos e estoques facilita esse processo, visto que as equações são inseridas para cada relação.

Com isso tem-se, então, o modelo para simulação encerrando as etapas de modelagem. A simulação permitirá explorar o impacto de determinadas ações no sistema.

\section{Simulação e análise}

A etapa de simulação propriamente é precedida pela certificação de utilidade do modelo. Um conjunto de testes, que são abordados com detalhes por Forrester \& Senge (1980), pode ser utilizado para aumentar a confiança na utilidade do modelo. Nessa pesquisa, são realizados testes de reprodução de comportamento que visam verificar a coerência e capacidade da estrutura do modelo em reproduzir o comportamento esperado.

Para tal, são adotados modelos gráficos de referência para a efetivação do teste de reprodução de comportamento. Esses modelos gráficos são padrões de comportamento tipicamente observados em sistemas análogos ou em aspectos específicos. Esses modelos são brevemente explanados a seguir:

(i) Comportamento da evolução de "produtos comercializados": Esse padrão gráfico representado pela "curva em onda" é utilizado por Sterman (2000) e Kazemi et al. (2011) na visualização das diferentes etapas do ciclo de vida de um produto no mercado. A consideração de que o comportamento favorável do sistema consiste no aumento da taxa de comercialização no tempo tem fundamento na análise desse comportamento.

(ii) Comportamento da dinâmica de "consumidores efetivos": O espaço amostral que determina a quantidade de consumidores efetivados pela aquisição (comercialização) do produto apresenta um comportamento, nos seus estágios iniciais, semelhante à evolução dos produtos comercializados. Contudo o montante total de consumidores efetivados apresenta acelerações e desacelerações, mas não apresenta reduções em função da descrição do sistema não contemplar eventuais devoluções de produtos. Esse padrão de referência também é empregado por Sterman (2000) e Kazemi et al. (2011). (iii) Comportamento da dinâmica de "consumidores potenciais": Conforme Sterman (2000) e Kazemi et al. (2011), o comportamento do volume de consumidores potenciais tem uma redução à medida que o volume de consumidores efetivos aumenta. Pela estrutura definida ao sistema, os consumidores potenciais são convertidos em consumidores efetivos em uma taxa específica do instante no tempo.

(iv) Comportamento do "conhecimento acumulado": A estrutura que representa a evolução do conhecimento acumulado insere as ações de controle correspondentes à gestão do conhecimento. É expressa por um ciclo de realimentação negativo e, como tal, é caracterizado graficamente por um crescimento orientado a um valor objetivo.

A Figura 5 apresenta os comportamentos simulados. Nessa apresentação, foram utilizadas as mesmas referências ordinais que a descrição do comportamento, exposta anteriormente. Para os testes, o modelo foi dividido em partes isoladas de modo conveniente para a verificação de relações específicas entre fatores. Ressalta-se que o foco dessa análise comparativa está no padrão de comportamento. Amplitudes ou uma distribuição diferenciada no horizonte de simulação são devidas aos valores específicos das constantes e variáveis.

Duas importantes considerações são realizadas nesse estágio da pesquisa: (i) observando que o modelo construído reproduz o comportamento esperado em certos aspectos de interesse esse modelo é considerado útil para essa análise e (ii) como o estado atual (valores) das constantes e variáveis do modelo proporcionou a reprodução do comportamento, o modelo também é considerado calibrado para a amplitude da análise desejada.

\subsection{Projeção de cenários}

A simulação computacional utilizando o modelo quantitativo permite analisar o comportamento de um sistema e testar ações diante de seu desempenho em um "mundo virtual" (Sterman, 2000), ou seja, permite estimar o impacto da alteração de algumas variáveis na saída do sistema. Reproduz o comportamento do sistema integrado, com todas as partes associadas e, por isso, associando os diferentes comportamentos das partes isoladas do sistema. Com isso, são projetados cenários de difícil intuição diante das não linearidades e realimentações de informações. É nesse sentido que a ferramenta computacional auxilia a compreensão na análise de sistemas complexos.

A projeção dos cenários utilizou o método de integração de "Euler" e, conforme o recomendado por Forrester (1961), o intervalo de integração (dt) foi 
(i)

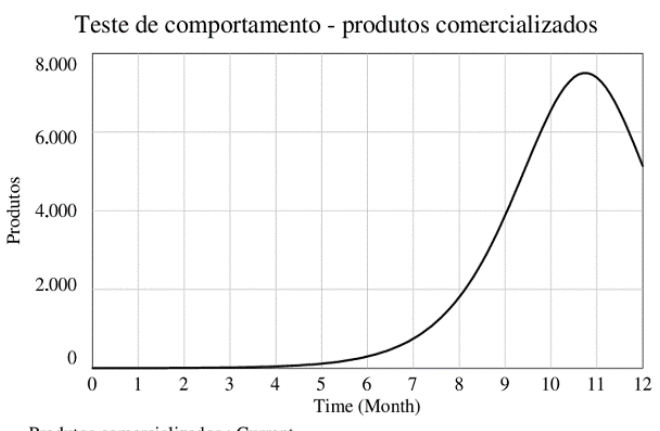

(iii)

Teste de comportamento - consumidores potenciais

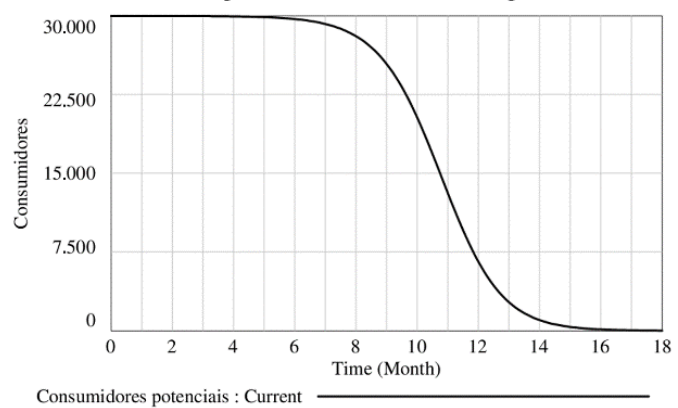

(ii)

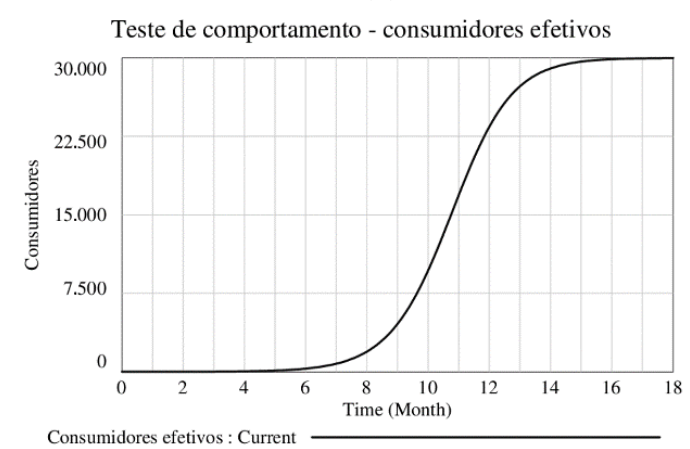

(iv)

Teste de comportamento - Conhecimento acumulado

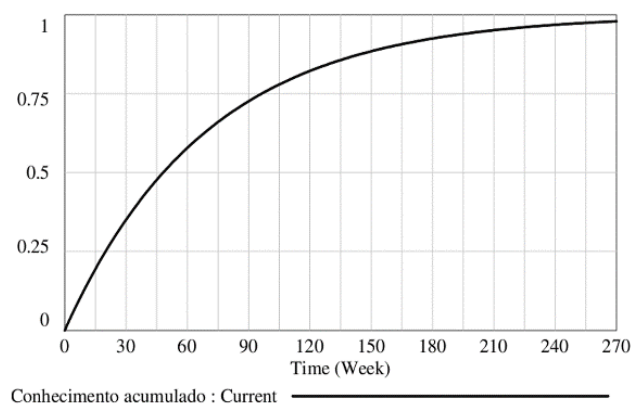

Figura 5. Comportamento apresentado nos testes do modelo. Fonte: Elaborado pelos autores.

utilizado como $1 / 5$ do valor da menor constante de tempo em cada configuração de simulação. A Figura 6 apresenta especificamente o comportamento gráfico da quantidade de produtos comercializados no horizonte de tempo, ou seja, a curva que representa o ciclo de vida do produto. O comportamento desta variável foi adotado como referência para a análise, uma vez que muitas ações táticas na comercialização dos produtos são estabelecidas tomando-a como referência.

Essa simulação possibilita observar que, quando a quantidade de unidades de produtos comercializados inicia uma redução (o estágio de declínio do ciclo de vida do produto), um novo comportamento crescente é estabelecido. Isso pode ser explicado tendo em vista que a geração de conhecimento externo influencia o aumento dos consumidores potenciais devido ao seu reflexo no surgimento de novas aplicações. A Figura 7 expõe o comportamento gráfico dos consumidores potenciais. A taxa de adesão também aumenta gradativamente até que o crescimento dos consumidores potenciais tenha uma redução pela conversão desses consumidores em efetivos. Contudo, uma nova elevação é observada, visto que, dado o tempo de vida do produto, os consumidores já efetivados voltam à condição de consumidores potenciais. Ou seja, são determinantes nesse comportamento tanto a abertura de novos mercados como a reincidência dos consumidores efetivos após o tempo de vida do produto.

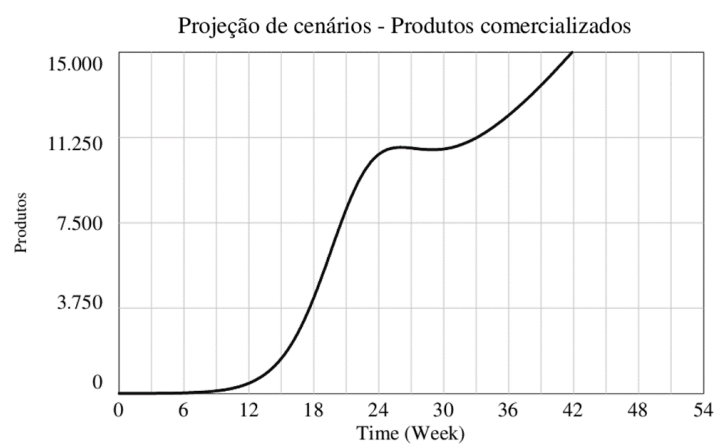

Produtos comercializados : Cenário_6 - graficos 1 e 2 -

Figura 6. Produtos comercializados. Fonte: Elaborado pelos autores.

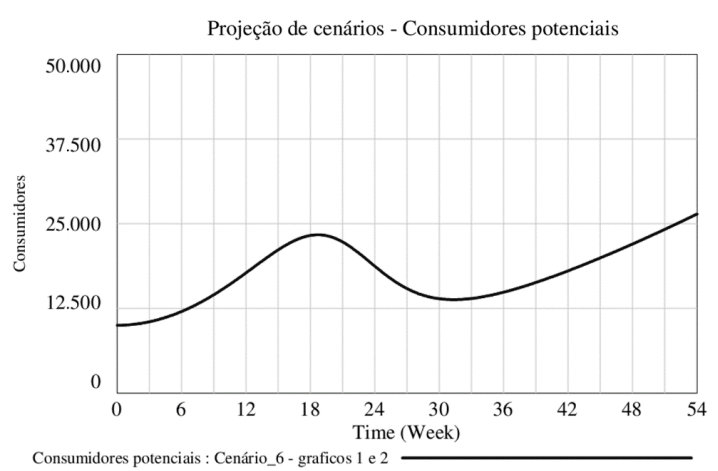

Figura 7. Comportamento gráfico de "Consumidores potenciais". Fonte: Elaborado pelos autores. 


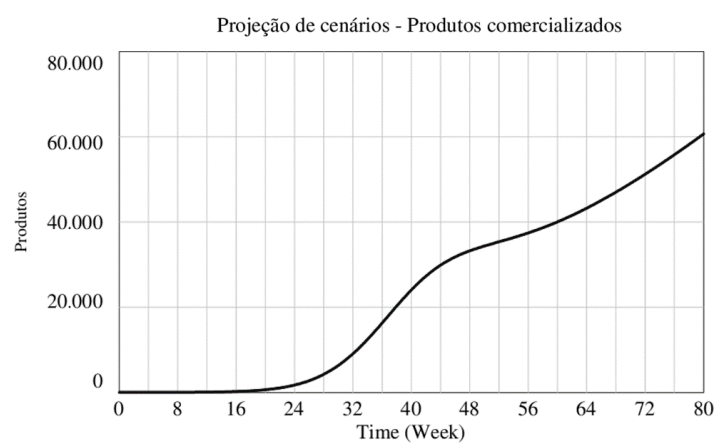

Produtos comercializados : Cenário_6 - graficos 3

Figura 8. Produtos comercializados (acréscimo de concorrentes). Fonte: Elaborado pelos autores.

Buscando subsídios para a análise, o modelo foi configurado para uma condição que representa um mercado com maior número de possíveis concorrentes para a mesma quantidade de conteúdo compartilhado. Nessa condição, comparativamente à anterior, o efeito da diferenciação do valor na satisfação do consumidor é reduzido em função da maior geração de valor pelo mercado. Nesse sentido, a taxa de adesão permanece inicialmente com valor reduzido e, como mostra a Figura 8, o início da comercialização dos produtos em um instante posterior. A influência nessa taxa também ameniza a quantidade de produtos comercializados. O desempenho do sistema para a geração do valor é reduzido podendo também ocasionar um comportamento oscilatório.

Nesse contexto, o surgimento de novas aplicações continua a contribuir com o acréscimo de potenciais consumidores. Isto mantém a tendência de crescimento da comercialização de produtos. Entretanto, se ressalta que esses comportamentos se referem somente ao contexto descrito que pode ser expandido e detalhado. Indica a possibilidade de correlação de fatores como o "tipo de conteúdo" (desde elementos conceituais até informações detalhadas e protótipos) envolvido na ação de compartilhamento, complementando ainda mais o contexto. Em termos de ações, o foco da efetivação da inovação para a diferenciação do valor.

\section{Conclusões}

Esta investigação foi conduzida abordando e representando o fenômeno da inovação aberta. O objetivo que orientou esta pesquisa consiste em: evidenciar a influência da (i) geração de conhecimento externo e da (ii) entrada de novos concorrentes na taxa de comercialização de produtos ao longo do tempo.

Como principal resultado, foi obtido um modelo para simulação computacional do fenômeno. Pelo comportamento projetado, com os fatores utilizados neste estudo, puderam ser analisados alguns aspectos da influência de ambas as variáveis na curva do ciclo de vida do produto. Os cenários gerados evidenciaram, para a configuração específica do modelo, que a geração de conhecimento externo pode prover a aceleração do processo de inovação. Essa aceleração promove a diferenciação do valor para a satisfação do consumidor; também a abertura de novos mercados pode promover maior visibilidade do produto. Com isso há uma evolução crescente da quantidade de produtos consumidos no tempo. Porém, a entrada de novos concorrentes pode ter uma ação de retardamento e abreviação deste crescimento.

As experiências conduzidas no modelo, de modo geral, mostram que, em um contexto de inovação aberta, as ações relativas à dinâmica da evolução desse produto no mercado, quando utilizam a avaliação e análise do ciclo de vida do produto, fatores dinâmicos específicos do mercado precisam ser considerados nesse contexto. Mostra também que a abertura do processo de inovação pode conduzir a contextos favoráveis quanto à comercialização do produto, ou seja, mesmo com a ocorrência de entrada de novos concorrentes, a taxa de comercialização de produtos pode ter um comportamento de aumento.

Os resultados deste trabalho indicam que o avanço dos estudos pode concentrar-se na exploração detalhada dos demais fatores que influem na dinâmica de potenciais entrantes e de como efetivar o conhecimento adquirido externamente em inovação no produto. Uma possibilidade está na correlação do tipo de conteúdo no contexto descrito.

Ainda, como resultados intermediários aos objetivos específicos da pesquisa, foram construídos (i) o diagrama de causalidade relacionando os principais fatores que descrevem conceitualmente o contexto. Também (ii) um diagrama estrutural relacionando seis estoques, sendo: concorrentes, consumidores potenciais, consumidores efetivos, novas aplicações, conhecimento acumulado e valor específico oferecido. Esses resultados intermediários viabilizaram o processo de simulação, mas não menos importante, a adoção de um processo estruturado para a caracterização do modelo pode contribuir com a facilidade de compreensão e a confiança no modelo. Assim, outra sugestão de futuras abordagens está na exploração de métodos complementares adequados à metodologia Dinâmica de Sistemas.

As limitações dessa pesquisa podem ser segmentadas em termos de generalizações e simplificações. O conteúdo compartilhado não diferencia informações de conteúdo físico como protótipos. Também, todas as variáveis denominadas no padrão de o "efeito de (variável de causa) em (variável de efeito)" simplificam muitas cadeias de causa e efeito. Ou seja, correspondem às simplificadas do modelo. Nessas também são adotadas relações de linearidades que podem não representar o comportamento real desses efeitos.

A principal contribuição deste trabalho está na possibilidade de calibração do modelo para contextos específicos. Os valores das constantes e variáveis utilizadas para esta simulação e análise são estimados 
observando uma coerência lógica. A validade da análise é assegurada, visto que os cenários comparados são gerados com os valores das constantes e variáveis mantidos inalterados, salvo as ações que diferenciam os cenários. Contudo, o modelo calibrado pode se fazer útil para gestores em uma aplicação direta ou para apoio a uma compreensão mais aprofundada de contextos específicos.

\section{Referências}

Amit, R., \& Zott, C. (2001). Value creation in e-business. Strategic Management Journal, 22(6-7), 493-520. http:// dx.doi.org/10.1002/smj.187.

Batzias, F. A., \& Siontorou, C. G. (2012). Creating a specific domain ontology for supporting R\&D in the science-based sector - The case of biosensors. Expert Systems with Applications, 39(11), 9994-10015. http:// dx.doi.org/10.1016/j.eswa.2012.01.216.

Chesbrough, H. W. (2006). Open Innovation: the new imperative for creating and profiting from technology. Boston: Harvard Business School.

Chesbrough, H. W., Vanhaverbeke, W., \& West, J., editors. (2006). Open innovation: researching a new paradigm. Oxford University Press.

Chesbrough, H., \& Appleyard, M. M. (2007). Open innovation and strategy. California Management Review, 50(1), 57-76. http://dx.doi.org/10.2307/41166416.

Chiaroni, D., Chiesa, V., \& Frattini, F. (2010). Unravelling the process from closed to open innovation: evidence from mature, asset-intensive industries. Research Management, 40(3), 222-245.

Confederação Nacional da Indústria - CNI. (2009). Conclusões do $4^{\circ}$ encontro nacional da indústria: prioridades e recomendações (2011-2014). Brasília. 60 p. Recuperado em 18 de maio de 2014, de http://arquivos. portaldaindustria.com.br/imgs/ENAI_09_CADERNOCONCLUSES_WEB.pdf.

Coyle, R. G. (1996). System dynamics modelling: a practical approach. London: Chapman \& Hall. 432 p.

Dahlander, L., \& Gann, D. M. (2010). How open is innovation? Research Policy, 39(6), 699-709. http:// dx.doi.org/10.1016/j.respol.2010.01.013.

Dumas, M., La Rosa, M., Mendling, J., \& Reijers, H. A. (2013). Fundamentals of business process management. Berlin: Springer. $399 \mathrm{p}$.

Enkel, E., Gassmann, O., \& Chesbrough, H. (2009). W. Open R\&D and Open Innovation: exploring the phenomenon. $R$ \& D Management, 39(4), 311-316. http://dx.doi.org/10.1111/j.1467-9310.2009.00570.x.

Forrester, J. W. (1961). Industrial dynamics. Massachusetts: Massachusetts Institute of Technology Press. 464 p.

Forrester, J. W., \& Senge, P. M. (1980). Test for building confidence in system dynamics models. TIMS Studies in the Management Sciences, (14), 209-228.
Huizingh, E. K. (2011). Open innovation: state of the art and future perspectives. Technovation, 31(1), 2-9. http://dx.doi.org/10.1016/j.technovation.2010.10.002.

Jackson, M. C. (2007). Systems thinking: creative holism for managers. Chichester: John Wiley \& Sons. 376 p.

Jiao, J., \& Tseng, M. M. (1999). A requirement management database system for product definition. Integrated Manufacturing Systems, 10(3), 126-131. http://dx.doi. org/10.1108/09576069910264402.

Kazemi, M. A. A., Eshlaghy, A. T., \& Tavasoli, S. (2011). Developing the product strategy via product life cycle simulation according to the system dynamics approach. Applied Mathematical Sciences, 5(17), 845-862.

Kotonya, G., \& Sommerville, I. (2000). Requirements engineering: process and techniques. Chichester: John Wiley \& Sons.

Labuschagne, A. C., Brent, A. C., \& van Erck, R. P. G. (2005). Assessing the sustainability performances of industries. Journal of Cleaner Production, 13(4), 373385. http://dx.doi.org/10.1016/j.jclepro.2003.10.007.

Lichtenthaler, U. (2011). Open innovation: past research, current debates, and future directions. The Academy of Management Perspectives, 25(1), 75-93. http://dx.doi. org/10.5465/AMP.2011.59198451.

Oculus VR. Recuperado em 20 de maio de 2014, de http:// www.oculusvr.com.

Organization for Economic Co-operation and Development - OECD. (2007). Oslo manual: proposed guideline for collecting and interpreting technological innovation data. Paris.

Organization for Economic Co-operation and Development - OECD. (2010). The OECD Innovation Strategy: getting a head start on tomorrow. Paris.

Sanches, P. L. B., \& Machado, A. G. C. (2014). Estratégias de inovação sob a perspectiva da Resourced-Based View: análise e evidências em empresas de base tecnológica. Gestão \& Produção, 21(1), 125-141. http://dx.doi. org/10.1590/S0104-530X2014005000005.

Sterman, J. D. (2000). Business dynamics: systems thinking and modeling for a complex world. McGraw-Hill. $982 \mathrm{p}$.

Tálamo, J. R., \& Carvalho, M. M. (2010). Redes de cooperação com foco em inovação: um estudo exploratório. Gestão \& Produção, 17(4), 747-760. http://dx.doi.org/10.1590/ S0104-530X2010000400009.

Tidd, J., Bessant, J., \& Pavitt, K. (2005). Gestão da inovação (3. ed.) Porto Alegre: Bookman. $600 \mathrm{p}$.

Van de Vrande, V., De Jong, J. P. J., Vanhaverbeke, W., \& De Rochemont, M. (2009). Open innovation in SMEs: trends, motives and management challenges. Technovation, 29(6-7), 423-437. http://dx.doi.org/10.1016/j. technovation.2008.10.001. 